\title{
Aplastic Anemia in a Patient with Cronkhite-Canada Syndrome
}

\author{
Keisuke Kidoguchi ${ }^{1}$, Yasushi Kubota ${ }^{1,2}$, Shun Fujimoto ${ }^{3}$, Yasuhisa Sakata ${ }^{3}$, \\ Haruna Kizuka-Sano ${ }^{1}$, Kyosuke Yamaguchi ${ }^{1}$, Hiroshi Ureshino ${ }^{1}$, Hiroo Katsuya ${ }^{1}$, \\ Toshihiko Ando ${ }^{1}$, Motohiro Esaki ${ }^{3}$ and Shinya Kimura ${ }^{1}$
}

\begin{abstract}
:
Cronkhite-Canada syndrome (CCS) is a rare polyposis disorder accompanied by alopecia and onychodystrophy. A 63-year-old man with a history of CCS and repeated embolism developed progressive thrombocytopenia and mild anemia. Laboratory testing, a bone marrow examination, and magnetic resonance imaging of the spine resulted in a diagnosis of concurrent aplastic anemia (AA). Paroxysmal nocturnal hemoglobinuria $(\mathrm{PNH})$-type cells were detected in a peripheral blood specimen. In addition, human leukocyte antigen (HLA) included DRB1*15:01 and DRB1*15:02. Mesalazine was discontinued in consideration of possible drug-induced pancytopenia. Immunosuppressive therapy ameliorated both the gastrointestinal symptoms of CCS and pancytopenia. A common autoimmune abnormality might underlie both CCS and AA.
\end{abstract}

Key words: Cronkhite-Canada syndrome, aplastic anemia, paroxysmal nocturnal hemoglobinuria, human leukocyte antigen, autoimmune disorder

(Intern Med 60: 1601-1605, 2021)

(DOI: 10.2169/internalmedicine.6468-20)

\section{Introduction}

Cronkhite-Canada syndrome (CCS) is a rare, noninherited gastrointestinal polyposis syndrome first described by Cronkhite and Canada in 1955 (1). Typical characteristics are generalized polyposis, pigmentation, alopecia, and onychodystrophy. Individuals of Asian descent (including Japanese) are the most commonly affected, with a median age at the onset of 59 years old (2).

This rare disease is considered to involve multiple causative factors, but none are definitive, and none have been confirmed in large-scale studies (3). An autoimmune etiology is plausible, as some cases of CCS are associated with antinuclear antibodies and show positive immunostaining for immunoglobulin $(\mathrm{Ig}) \mathrm{G} 4(4,5)$. Aplastic anemia (AA) is also thought to be caused by immune-mediated hematopoietic suppression $(6,7)$.
We herein report a case of CCS with AA complicated by recurrent thromboembolic events. To the best of our knowledge, this is the first report to describe AA concomitant with CCS.

\section{Case Report}

A 57-year-old man visited a local hospital complaining of right lower leg pain, malaise, and dyspnea. Computed tomography revealed deep vein thrombosis in the right lower leg and bilateral pulmonary emboli. Anticoagulant therapy was initiated and successfully eliminated the emboli. During hospitalization, occult blood was detected in stools and colonoscopy was therefore performed. A tentative diagnosis of ulcerative colitis (UC) was made, and mesalazine was commenced.

However, one month later, atypical manifestations of dysgeusia, alopecia, pruritis, and peripheral neuropathy

\footnotetext{
${ }^{1}$ Division of Hematology, Respiratory Medicine and Oncology, Department of Internal Medicine, Faculty of Medicine, Saga University, Japan, ${ }^{2}$ Department of Transfusion Medicine, Saga University Hospital, Japan and ${ }^{3}$ Division of Gastroenterology, Department of Internal Medicine, Faculty of Medicine, Saga University, Japan

Received for publication October 8, 2020; Accepted for publication November 2, 2020

Correspondence to Dr. Yasushi Kubota, kubotay@cc.saga-u.ac.jp
} 


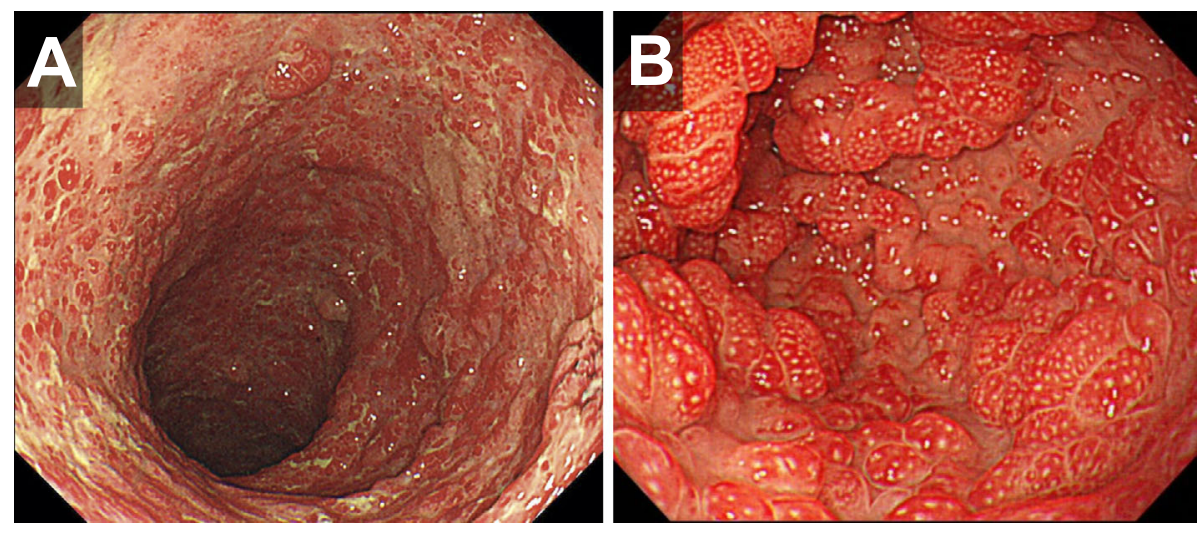

Figure 1. Endoscopic findings. A) Small villous polyposis throughout the lower gastrointestinal tract. B) Numerous inflammatory polyps proliferating in the lining of the stomach.

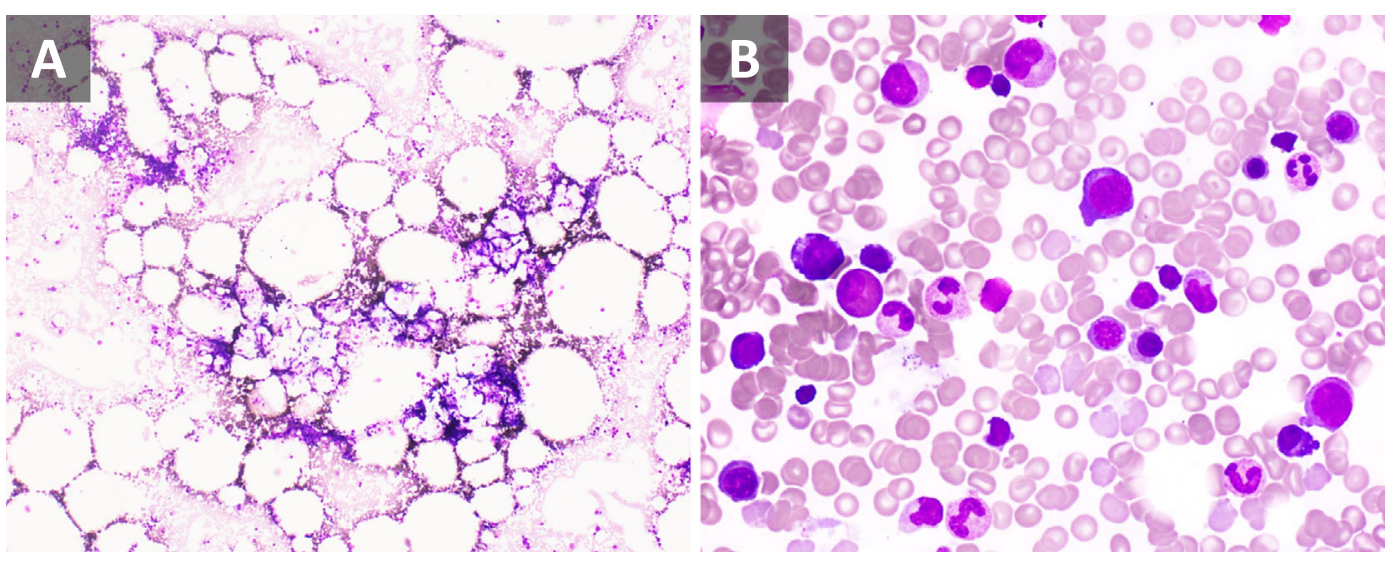

Figure 2. Bone marrow examination findings. May-Grunwald Giemsa staining of a bone marrow smear. Hypoplastic marrow without proliferation of immature hematopoietic cells. No dysplasia is seen. Magnification, $\times 40$ (A), $\times 400(B)$.

emerged. Unresolved diarrhea and hypo-albuminemia also appeared. Mesalazine was discontinued as a possible cause of adverse effects, especially concerning liver dysfunction. Repeated colonoscopy and esophagogastroduodenoscopy revealed an edematous and reddish mucosa and numerous polypoid lesions (Fig. 1). A biopsy specimen from the terminal ileum showed infiltration of the edematous mucosa by various inflammatory cells. The diagnosis was changed from UC to a definitive diagnosis of CCS according to the clinical manifestations and pathological findings.

The patient received prednisolone (PSL) at $60 \mathrm{mg} / \mathrm{day}$, which gradually mitigated the bloody diarrhea and dysgeusia. Repeated endoscopy revealed reduced numbers of polyps in both the upper and lower gastrointestinal tract. Annual follow-up endoscopy was scheduled. PSL was deliberately tapered, but decreasing the dose to $<4 \mathrm{mg} /$ day led to difficulty controlling the gastrointestinal symptoms. Eventually, to avoid side effects of corticosteroid therapy, mesalazine was added as combination therapy for CCS when PSL was reduced to $15 \mathrm{mg} /$ day. PSL was finally tapered to $2 \mathrm{mg} /$ day in combination with mesalazine at 2,000 mg/day, with no recurrence of liver dysfunction.

Two years later, the patient developed sudden left-sided paralysis and aphasia, accompanied by dizziness. Magnetic resonance imaging (MRI) of the head revealed a pale, nodular lesion in the dentate nucleus of the right cerebellum. In addition, multiple cerebral infarctions were noted in the left frontal lobe and cerebral hemispheres and were considered signs of previous, repeated asymptomatic strokes. Anticoagulant therapy was switched from warfarin to edoxaban.

Five years after the CCS diagnosis, a routine visit revealed progressive thrombocytopenia, neutropenia, and mild anemia. The laboratory parameters were as follows: hemoglobin $(\mathrm{Hb}), 9.6 \mathrm{~g} / \mathrm{dL}$ [normal range (NR) 13.7-16.8 g/dL]; reticulocyte count, $51.1 \times 10^{9} / \mathrm{L}\left(\mathrm{NR} 29.0-92.3 \times 10^{9} / \mathrm{L}\right)$; white blood cell (WBC) count, $3.6 \times 10^{9} / \mathrm{L}\left(\mathrm{NR} 3.3-8.6 \times 10^{9} / \mathrm{L}\right)$ with $23.1 \%$ neutrophils; $69.7 \%$ lymphocytes; $6.1 \%$ monocytes; and no blasts; and a platelet count of $20.0 \times 10^{9} / \mathrm{L}$ (NR 158$\left.348 \times 10^{9} / \mathrm{L}\right)$. The platelet count fell sharply over a six-month period, preceding decreases in the $\mathrm{Hb}$ value and neutrophil count. Flow cytometry detected paroxysmal nocturnal hemoglobinuria (PNH) clones among granulocytes $(1.041 \%)$ and red blood cells $(0.387 \%)$. A bone marrow examination revealed hypoplastic marrow with no signs of hematological malignancy, such as leukemia or myelodysplastic syndrome (Fig. 2). Both T1- and T2-weighted MRI of the spine 


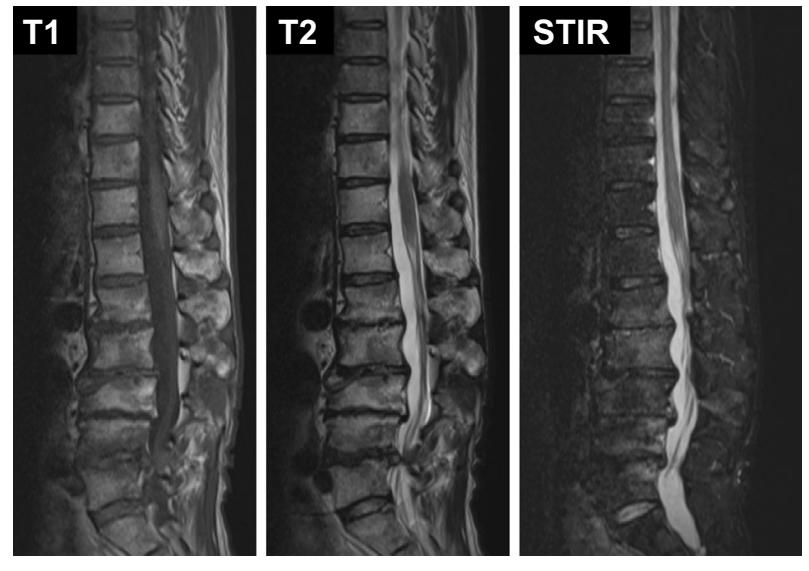

Figure 3. MRI findings of the spine. MRI of the spine reveals hyperintensity of the bone marrow on both T1- and T2-weighted imaging. The hyperintense region is hypointense on shorttau inversion recovery MRI.

showed mainly high signal intensity (SI) areas. These areas were depicted as low SI on short-tau inversion recovery (STIR) imaging, suggesting that the bone marrow had been completely replaced by fat (Fig. 3). Taken together, these findings led to a diagnosis of moderate AA that does not require a blood transfusion at all. In addition, we identified haplotypes of human leukocyte antigen (HLA) as DRB $1 * 15$ : 01 and DRB1*15:02, which are common in Japanese patients with AA (Table).

As the patient had suffered repeated thrombosis, we also evaluated his thrombotic status. A lupus anticoagulant (LAC) panel yielded a positive result for dilute Russell's viper venom time but a negative result for the activated partial thromboplastin time. Other risk factors associated with thromboembolism, including protein $\mathrm{C}$ antigen, protein $\mathrm{C}$ activity, protein $\mathrm{S}$ activity, and serum anti-phospholipid antibodies, were within normal ranges.

The clinical course is shown in Fig. 4. Mesalazine (to treat CCS) was discontinued due to its potential risk of causing AA. The total administration period before discontinuation was 22 months. The patient subsequently received a standard dose of cyclosporine (CsA), with the trough concentration adjusted to achieve a target range of 150-250 ng/ $\mathrm{mL}$. Although the patient had briefly required platelet transfusion, the platelet counts gradually increased and were maintained at $>10 \times 10^{9} / \mathrm{L}$. At the same time, the WBC count increased gradually in the absence of granulocyte colonystimulating factor (G-CSF).

Despite these treatments, accompanying anemia slowly deteriorated. At 4 months after starting CsA, PSL was once again increased from $2 \mathrm{mg} /$ day to $20 \mathrm{mg}$ /day because of a flare of diarrhea. Reticulocytes subsequently increased. At five months after starting CsA, eltrombopag (EPAG) was commenced with the intention of ameliorating thrombocytopenia. At the time of writing, the patient remains free from blood transfusion on 3-drug combination therapy of PSL at $14 \mathrm{mg} /$ day, $\mathrm{CsA}$ at $275 \mathrm{mg} / \mathrm{day}$, and EPAG at 75
Table. Human Leukocyte Antigen Haplotype (HLA) of the Patient.

\begin{tabular}{lcc}
\hline \multicolumn{1}{c}{ Locus } & Allele & Allele \\
\hline HLA-A & $24: 02$ & $26: 02$ \\
HLA-B & $35: 01$ & $52: 01$ \\
HLA-C & $03: 03$ & $12: 02$ \\
\hline HLA-DRB1 & $15: 01$ & $15: 02$ \\
HLA-DQB1 & $06: 01$ & $06: 02$ \\
HLA-DPB1 & $05: 01$ & $09: 01$ \\
\hline
\end{tabular}

$\mathrm{mg} /$ day without recurrence of diarrhea or any other clinical symptoms of CCS.

\section{Discussion}

We encountered a case of AA occurring in a patient with CCS treated using mesalazine. Symptoms were improved by immunosuppressive therapy using CsA. AA occurring after mesalazine administration for inflammatory bowel disease (IBD) has been reported as drug-induced pancytopenia, known as "mesalazine-induced AA." However, in such cases, PNH-type blood cells are often detected, as in our case, suggesting the involvement of immunological mechanisms.

The established treatment for CCS is immunosuppressive therapy $(3,8,9)$. Corticosteroids and CsA are the drugs of choice, with or without mesalazine. Corticosteroids are critical for treating autoimmune disorders, but steroid-sparing strategies are often used to avoid the side effects (10). Mesalazine is widely used to treat IBDs, such as UC and Crohn's disease. The side effects of mesalazine include hematologic disorders such as agranulocytosis, hemolytic anemia, and AA. Late-onset AA is a rare but potentially fatal adverse effect (11-16). The onset of mesalazine-induced AA varies from months to years, but a one-year period is usually required to diagnose mesalazine-induced AA. The treatment for mesalazine-induced AA is, of course, drug discontinuation. However, bone marrow recovery is not a rapid process, and additional supportive treatment including GCSF is sometimes needed $(11,12)$. Four case reports have described utilizing antilymphocyte/antithymocyte globulin therapy (as a monotherapy or combination with CsA) $(11,13,15)$, two of which were treated successfully $(11,14)$. Previous case reports have suggested these treatment strategies, but unfortunately, patients frequently develop severe infections due to profound neutropenia, often leading to death $(13,16)$.

Drug-induced AA is a consequence of chemical and physical damage to bone marrow and is treated by supportive care, including blood transfusions and hematopoietic growth factors (17). In the present case, we started immunosuppressive therapy using CsA three days after the discontinuation of mesalazine, due to the severity of thrombocy- 


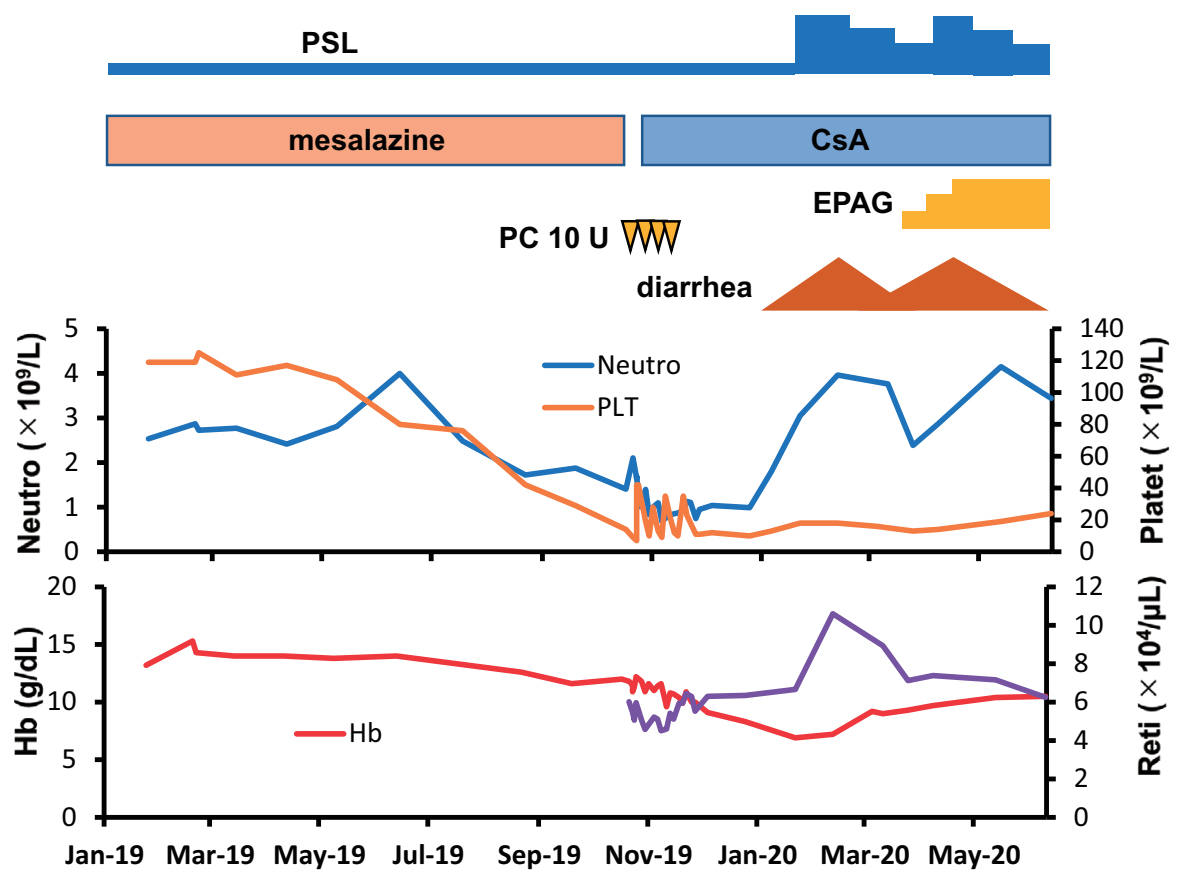

Figure 4. Clinical course. Due to the progressive thrombocytopenia and mild anemia, mesalazine was discontinued. Cyclosporine was initiated, and the platelets recovered with transient platelet transfusions. PSL: prednisolone, CsA: cyclosporine, PC: platelet concentrate, EPAG: eltrombopag, Neutro: neutrophils, Hb: hemoglobin, Reti: reticulocytes

topenia and neutropenia. Although insufficient time was available to observe bone marrow recovery with mesalazine discontinuation alone, hematological improvement was slow even after the initiation of immunosuppressive therapy. Furthermore, a flow cytometry analysis of peripheral blood from the present patient showed increased percentages of PNH-type cells $(18,19)$, which are regarded as a marker for immune-mediated bone marrow failure. Studies of PNH-type cells from AA patients complicated by UC have shown a high prevalence of increased PNH-type cells, even among mesalazine-treated patients, suggesting that AA associated with UC has an immune-mediated mechanism, and mesalazine itself may have been unrelated to the development of AA $(20,21)$.

Specific HLA profiles are linked to autoimmune disorders (22-24). HLA-DRB1*15:01 is more common in AA $(25,26)$, and patients showing DRB1*15:01 are more likely to improve in response to CsA (27). In Japan, the frequency of HLA DRB1*15:01 and DRB1*15:02 is significantly higher in patients with AA than in healthy controls (28). Of interest, HLA-DRB1*15:02 has been reported to show a strong association with UC among Japanese patients (29). Nakagawa et al. investigated HLA profiles in 14 AA patients with UC, and six patients proved positive for DRB1*15:02. In addition, another major DR15 allele, DRB1*15:01, was confirmed in four patients. Two patients had both DRB1*15:01 and DRB1*15:02. These results suggest a common immune pathophysiology between AA and UC (20). Only one case report described HLA-A/B typing of a patient with CCS, but the HLA DR loci were not de- scribed (30). Interestingly, the present patient had both HLA DRB $1 * 15: 01$ and DRB $1 * 15: 02$. As CCS is considered an IBD-related disease, further investigation is warranted to elucidate the significance of HLA and its association with CCS.

Repeated thromboembolism is another concern. Several case reports have described the cooccurrence of thrombotic event with CCS (31-33). Possible causes of thrombosis are concomitant malignancy $(31,32)$ and sepsis $(33)$, but these factors were not confirmed in our case. Corticosteroid, which is a key drug for the treatment of CCS, is a wellknown predisposing factor for thromboembolism (34). Although corticosteroid might have induced cerebellar infarction in our case, regardless of anticoagulation with warfarin, his first thrombotic event occurred before the start of corticosteroid treatment for CCS. Corticosteroid was thus an unlikely cause of his first episode of thromboembolism (deep vein thrombosis in the right lower leg and bilateral pulmonary emboli). Because a LAC panel yielded a positive result for dilute Russell's viper venom time, the coagulant disorder might be more closely related to autoimmune abnormalities than coexisting comorbidities and corticosteroid use.

In conclusion, autoimmune abnormalities suggested by the presence of antinuclear antibodies, positive immunostaining for IgG4, and concurrent AA in the present case were considered plausible etiological factors for CCS. The efficacy of immunosuppressive therapy also supports this concept. Why most patients with CCS are Japanese is unclear. Racial variations in HLA profiles and their associations with CCS are of interest. Although we were unable to clearly dis- 
criminate idiopathic from mesalazine-induced AA in this case, a common autoimmune abnormality might underlie both CCS and AA.

The authors state that they have no Conflict of Interest (COI).

\section{References}

1. Cronkhite LW Jr., Canada WJ. Generalized gastrointestinal polyposis; an unusual syndrome of polyposis, pigmentation, alopecia and onychotrophia. N Engl J Med 252: 1011-1015, 1955.

2. Daniel ES, Ludwig SL, Lewin KJ, Ruprecht RM, Rajacich GM, Schwabe AD. The Cronkhite-Canada syndrome. An analysis of clinical and pathologic features and therapy in 55 patients. Medicine (Baltimore) 61: 293-309, 1982.

3. Watanabe C, Komoto S, Tomita K, et al. Endoscopic and clinical evaluation of treatment and prognosis of Cronkhite-Canada syndrome: a Japanese nationwide survey. J Gastroenterol 51: 327-336, 2016.

4. Ota S, Kasahara A, Tada S, et al. Cronkhite-Canada syndrome showing elevated levels of antinuclear and anticentromere antibody. Clin J Gastroenterol 8: 29-34, 2015.

5. Sweetser S, Ahlquist DA, Osborn NK, et al. Clinicopathologic features and treatment outcomes in Cronkhite-Canada syndrome: support for autoimmunity. Dig Dis Sci 57: 496-502, 2012.

6. Nakao S, Feng X, Sugimori C. Immune pathophysiology of aplastic anemia. Int J Hematol 82: 196-200, 2005.

7. Young NS, Calado RT, Scheinberg P. Current concepts in the pathophysiology and treatment of aplastic anemia. Blood 108: 2509-2519, 2006.

8. Schulte S, Kutting F, Mertens J, et al. Case report of patient with a Cronkhite-Canada syndrome: sustained remission after treatment with corticosteroids and mesalazine. BMC Gastroenterol 19: 36, 2019.

9. Yamakawa K, Yoshino T, Watanabe K, et al. Effectiveness of cyclosporine as a treatment for steroid-resistant Cronkhite-Canada syndrome; two case reports. BMC Gastroenterol 16: 123, 2016.

10. Mao EJ, Hyder SM, DeNucci TD, Fine S. A successful steroidsparing approach in Cronkhite-Canada syndrome. ACG Case Rep J 6: 1-4, 2019.

11. Otsubo H, Kaito K, Sekita T, Shimada T, Kobayashi M, Hosoya T. Mesalazine-associated severe aplastic anemia successfully treated with antithymocyte globulin, cyclosporine and granulocyte colonystimulating factor. Int J Hematol 68: 445-448, 1998.

12. Wyatt $S$, Joyner MV, Daneshmend TK. Filgrastim for mesalazineassociated neutropenia. Lancet 341: 1476, 1993.

13. Abboudi ZH, Marsh JC, Smith-Laing G, Gordon-Smith EC. Fatal aplastic anaemia after mesalazine. Lancet 343: 542, 1994.

14. Laidlaw ST, Reilly JT. Antilymphocyte globulin for mesalazineassociated aplastic anaemia. Lancet 343: 981-982, 1994.

15. Wiesen A, Wiesen J, Limaye S, Kaushik N. Mesalazine-induced aplastic anemia. Am J Gastroenterol 104: 1063, 2009.

16. Daneshmend TK. Mesalazine-associated thrombocytopenia. Lancet 337: 1297-1298, 1991.

17. Young NS. Aplastic anemia. N Engl J Med 379: 1643-1656, 2018.

18. Sugimori C, Chuhjo T, Feng X, et al. Minor population of CD55 CD59 blood cells predicts response to immunosuppressive therapy and prognosis in patients with aplastic anemia. Blood 107: 13081314, 2006.

19. Griscelli-Bennaceur A, Gluckman E, Scrobohaci ML, et al. Aplastic anemia and paroxysmal nocturnal hemoglobinuria: search for a pathogenetic link. Blood 85: 1354-63, 1995.

20. Nakagawa $\mathrm{N}$, Maruyama $\mathrm{H}$, Zaimoku $\mathrm{Y}$, et al. Evidence for a common immune pathophysiology in acquired aplastic anemia and ulcerative colitis. Blood 126: 2418, 2015.

21. Shimada T, Maeda T, Ishikawa M, et al. [Aplastic anemia complicated with ulcerative colitis]. Rinsho Ketsueki (Jpn J Clin Hematol) 53: 224-228, 2012 (in Japanese, Abstract in English).

22. Dendrou CA, Petersen J, Rossjohn J, Fugger L. HLA variation and disease. Nat Rev Immunol 18: 325-339, 2018.

23. Gough SC, Simmonds MJ. The HLA region and autoimmune disease: associations and mechanisms of action. Curr Genomics 8: 453-465, 2007.

24. Kular L, Liu Y, Ruhrmann S, et al. DNA methylation as a mediator of HLA-DRB1*15:01 and a protective variant in multiple sclerosis. Nat Commun 9: 2397, 2018.

25. Nimer SD, Ireland P, Meshkinpour A, Frane M. An increased HLA DR2 frequency is seen in aplastic anemia patients. Blood 84: 923-927, 1994.

26. Dhaliwal JS, Wong L, Kamaluddin MA, Yin LY, Murad S. Susceptibility to aplastic anemia is associated with HLA-DRB $1 * 1501$ in an aboriginal population in Sabah, Malaysia. Hum Immunol 72: 889-892, 2011.

27. Nakao S, Takamatsu H, Chuhjo T, et al. Identification of a specific HLA class II haplotype strongly associated with susceptibility to cyclosporine-dependent aplastic anemia. Blood 84: 4257-4261, 1994.

28. Sugimori C, Yamazaki H, Feng X, et al. Roles of DRB $1 * 1501$ and DRB1*1502 in the pathogenesis of aplastic anemia. Exp Hematol 35: 13-20, 2007.

29. Futami S, Aoyama N, Honsako Y, et al. HLA-DRB1*1502 allele, subtype of DR15, is associated with susceptibility to ulcerative colitis and its progression. Dig Dis Sci 40: 814-818, 1995.

30. Ali M, Weinstein J, Biempica L, Halpern A, Das KM. CronkhiteCanada syndrome: report of a case with bacteriologic, immunologic, and electron microscopic studies. Gastroenterology 79: 731-736, 1980.

31. Nemade NL, Shukla UB, Wagholikar GD. Cronkhite Canada syndrome complicated by pulmonary embolism - a case report. Int $\mathrm{J}$ Surg Case Rep 30: 17-22, 2017.

32. Takeuchi Y, Yoshikawa M, Tsukamoto N, Shiroi A, Hoshida Y, Enomoto Y, et al. Cronkhite-Canada syndrome with colon cancer, portal thrombosis, high titer of antinuclear antibodies, and membranous glomerulonephritis. J Gastroenterol 38: 791-795, 2003.

33. Hsu YQ, Loke SL. Cronkhite-Canada syndrome. Gastrointest Endosc 57: 917, 2003.

34. Johannesdottir SA, Horváth-Puhó E, Dekkers OM, et al. Use of glucocorticoids and risk of venous thromboembolism: a nationwide population-based case-control study. JAMA Intern Med 173: 743-752, 2013.

The Internal Medicine is an Open Access journal distributed under the Creative Commons Attribution-NonCommercial-NoDerivatives 4.0 International License. To view the details of this license, please visit (https://creativecommons.org/licenses/ by-nc-nd/4.0/).

(C) 2021 The Japanese Society of Internal Medicine Intern Med 60: 1601-1605, 2021 